\section{Superconducting Receiver Coils for Sodium Magnetic Resonance Imaging}

\author{
J. R. Miller,* K. Zhang, Q. Y. Ma, I. K. Mun, K. J. Jung, \\ J. Katz, D. W. Face, and D. J. Kountz
}

\begin{abstract}
We present the results from sodium magnetic resonance imaging (MRI) experiments using high-temperature superconducting (HTS) receiver coils. Sodium imaging has been shown to have great potential for the assessment of cell integrity but suffers from a substantially lower signal-to-noise ratio (SNR) than that of hydrogen imaging. The use of an HTS receiver coil was found to significantly increase the SNR relative to an equivalent copper receiver coil at room temperature. The SNR gains afforded by HTS coils can also be used to decrease the imaging time.
\end{abstract}

\section{INTRODUCTION}

The noise floor of conventional magnetic resonance imaging (MRI) systems is determined by the receiver coil noise for low frequency imaging and small fields of view (FOV). The signal-to-noise ratio (SNR) can be increased under these conditions by reducing the coil noise. High-temperature superconducting (HTS) coils below their critical temperature exhibit extremely low noise in the radio frequency (RF) range as a result of their low ac resistance. Some prior studies have focused on lowering the thermal noise of copper receiving coils by decreasing the operating temperature of the coil [1] and [2]. However, the Johnson noise power is proportional to the product of temperature and resistance. Copper cannot achieve the same minimum noise level in the RF range because the surface resistance of copper is orders of magnitude greater than high quality HTS material.

The imaging of sodium nuclei holds enormous potential as a diagnostic tool in distinguishing normal and damaged tissue. In some cases, sodium MRI has shown potential clinical advantages over hydrogen MRI, in particular with regard to early detection of certain brain disorders [3]. Much of the current work involving sodium is focused on multiple-quantum filtered (MQF) imaging [4], [5]. The major objective of these studies is to map the intracellular and extracellular sodium compartments [6]. The transmembrane sodium gradient is found to be sensitive to disease and consequently there is great interest in differentiating the sodium compartments. However, the low sensitivity of sodium has been the major obstacle to MQF imaging [7].

Previous studies have shown HTS coils to significantly increase the SNR relative to equivalent copper coils. Black et al. developed HTS coils for microscopy at $7 \mathrm{~T}$ and improved the SNR by about a factor

Manuscript received March 28, 1996; revised August 6, 1996. This work was supported by the Innovation Enhancement Fund (IEF) at Columbia University. Asterisk indicates corresponding author.

$*$ J. R. Miller is with the Department of Electrical Engineering, 1312 Mudd Building, Columbia University, New York, NY 10027 USA (e-mail: jrm33@columbia.edu).

K. Zhang, and Q. Y. Ma are with the Department of Electrical Engineering, Columbia University, New York, NY 10027 USA

I. K. Mun is with the Department of Radiology, Columbia University, New York, NY 10032 USA.

K. J. Jung is with the Department of Medicine, Columbia University, New York, NY 10032 USA.

J. Katz is with the Departments of Radiology and Medicine, Columbia University, New York, NY 10032 USA.

D. W. Face and D. J. Kountz are with DuPont, Wilmington, DE 19880 USA.

Publisher Item Identifier S 0018-9294(96)08983-5.

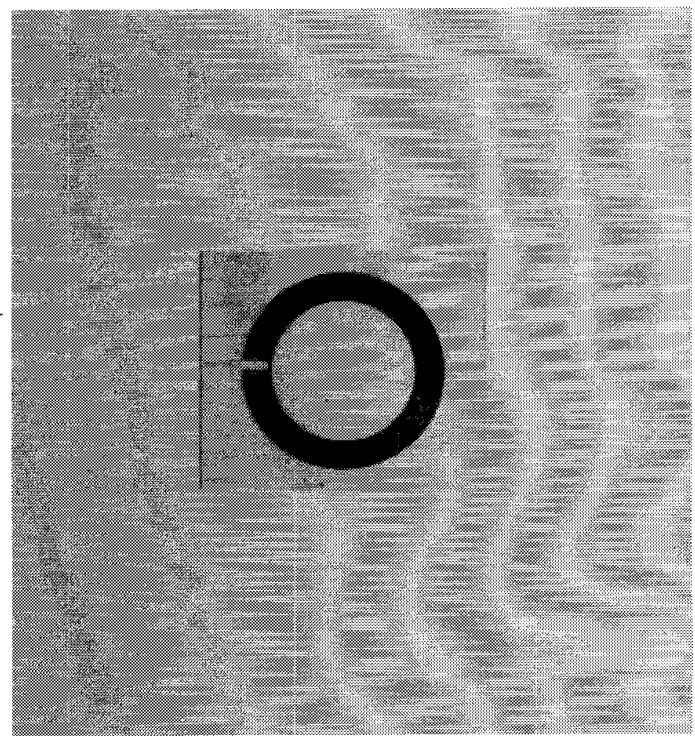

Fig. 1. A TBCCO superconducting receiver coil for sodium MRI.

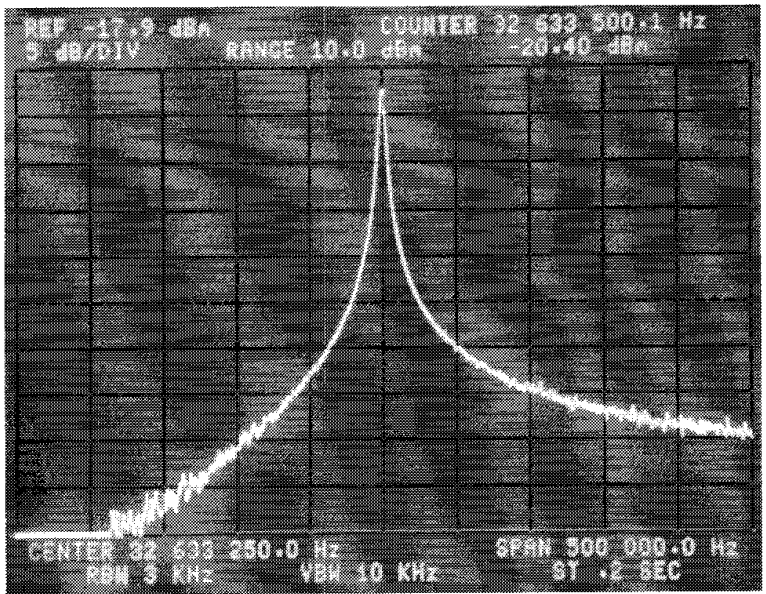

Fig. 2. Response of a sodium receiver coil fabricated from TBCCO. The $Q$ is about 37000 at a center frequency of about $33 \mathrm{MHz}$.

of 10 [8]. The same group made further refinements and reported that the SNR increased by a factor of 30 relative to a copper coil at room temperature [9]. More recently; a 9.4-T microscopy system that incorporates an HTS coil was demonstrated [10]. These studies have all focused on hydrogen imaging. The marginal signal levels of sodium provide the ideal situation for applying HTS receiver coils. Moreover, due to the low Lamor frequency of sodium, HTS coils can achieve SNR gains at high magnetic field strengths. In this paper, we describe the design of an HTS receiver coil system for sodium imaging and present the results of sodium imaging experiments.

\section{EXPERIMENTAL DESIGN AND METHODS}

\section{A. HTS Receiver Coil}

The HTS receiver coil used for this study was fabricated from a - $\mathrm{Tl}_{2} \mathrm{Ba}_{2} \mathrm{CaCu}_{2} \mathrm{O}_{8}$ (TBCCO) film on a $\mathrm{LaAlO}_{3}$ substrate [11]. The 


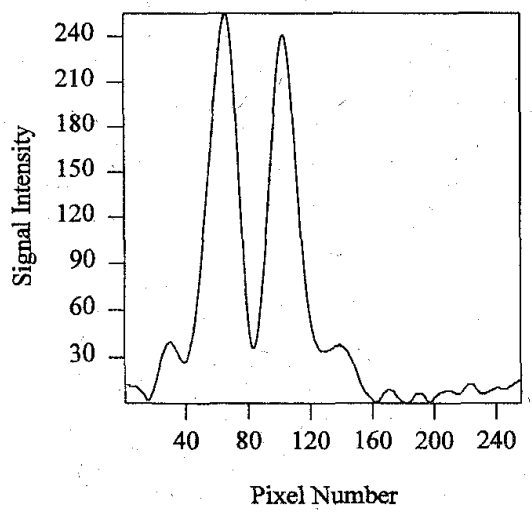

(a)

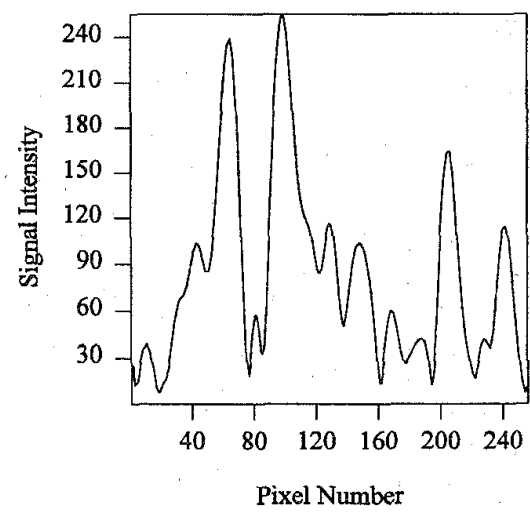

(b)

Fig. 3. Image profiles for: (a) an HTS coil and (b) a room temperature copper coil. Each profile consists of 256 pixels. The SNR is improved by a factor of ten with the HTS coil.

critical temperature $\left(T_{C}\right)$ of the film is about $105 \mathrm{~K}$. The coil design consists of a single coil inductor with interdigital capacitors between the turns of the inductor [12]. Dielectric loss is minimized in this design by the use of distributed capacitance which reduces the voltages associated with the fringing capacitances. The coil design is convenient from a fabrication perspective in that the design does not require a crossover which would be necessary for multiturn spiral coils. This avoids the complications associated with the fabrication of a multilevel structure. The films were fabricated using off-axis sputtering and patterned by ion milling. A Teflon film was then deposited after patterning to protect the coil from degradation. Fig. 1 shows a photograph of the HTS coil.

The coil was designed to resonate at the Larmor frequency of sodium in a 3-T field. The outer and inner diameters of the coil are 2.45 and $1.8 \mathrm{~cm}$, respectively. The coil dimensions were chosen to achieve a significant SNR gain factor. The inductor line widths are $100 \mu \mathrm{m}$ and the interdigital fingers are $33-\mu \mathrm{m}$ wide and spaced 22 $\mu \mathrm{m}$ apart.

\section{B. Copper Receiver Coil}

For the purpose of comparison a copper receiver coil was fabricated. As pointed out by van Heteran et al. [13], an ideal comparison would be between two coils of identical geometry with only the conductor material changed. However, a copper coil of identical geometry would exhibit very high conductor resistance and would therefore not be a fair comparison. A copper coil was therefore constructed which has wider windings but has a similar magnetic field pattern and FOV. The outer and inner diameters of the coil are 2.45 and $2.2 \mathrm{~cm}$, respectively. The copper coil consists of tightly wound 22-gauge enamel-covered solid copper wire and a fixed capacitance.

\section{Helium Cryostat}

The strong magnetic field produced by MRI magnets introduces vortex flux structures in the HTS material. These fluxoids tend to gather in regions of the material with grain boundaries. The motion of fluxoids is believed to be a dominant loss mechanism. The loss resulting from fluxoid motion is increased at temperatures near $T_{C}$ and can therefore be reduced by operating the superconductor at lower temperatures. Consequently, liquid helium is the preferred cryogen, permitting operation of the HTS coil well below $T_{C}$. The use of liquid helium, however, requires a vacuum insulated cryostat in order to achieve sufficiently low temperatures and maintain the helium boiloff rate at an acceptably low level. Most cryostats also incorporate a radiation shield to reduce the radiant heat flux. The metal content of these shields gives rise to eddy current losses and disturbs the homogeneity of the magnetic field.

A custom flow cryostat was designed and constructed which includes a nonmagnetic and nonconductive section in the critical region around the HTS coil and sample. The 6-in-long section is vacuum insulated with 1.5 -in-square polyvinylchloride (PVC). Sapphire provides thermal contact between the HTS coil and the copper cold stage, through which liquid helium flows. Sapphire was chosen for its excellent low temperature thermal properties and low dielectric loss. The vertical position of the sapphire can be adjusted to within $1 \mathrm{~mm}$ of the PVC wall or about $3 \mathrm{~mm}$ from the sample. This arrangement allows the HTS coil to be optimally positioned depending on where the region of interest is located.

The necessary connections to the HTS coil from the outside world are accomplished with two O-ring sealed feed-throughs. The first feed-through accommodates a $50 \Omega$ coaxial transmission line which is inductively coupled to the HTS coil. This coupling scheme preserves the SNR gain afforded by the HTS coil and provides an efficient way to match the input impedance of the preamplifier to the HTS coil [14]. The second feed-through is used for a tuning rod which provides a way of finely adjusting the resonant frequency of the HTS coil. Small adjustments are almost always necessary for each new sample because the electric and magnetic coupling of the HTS coil to the sample inevitably induces shifts in the resonant frequency.

The cryostat is equipped with a heater and platinum resistance thermometer, located in the copper cold stage. Platinum thermometers are capable of reliable operation in a high magnetic field environment. An external temperature controller maintains the HTS coil at $40 \mathrm{~K}$ $\pm 0.1 \mathrm{~K}$ via a nonmagnetic electronic feed-through.

\section{CoIl Measurements}

Coils are often characterized by a quality factor $(Q)$ which is a measure of the amount of energy stored compared to the losses in the coil. High $Q$ coils have reduced resistance which implies low Johnson noise and a higher SNR, assuming of course that coil noise is dominant. The $Q$ of the HTS coil and copper coil were determined by performing reflection measurements with a Hewlett-Packard 8753A network analyzer. The HTS coil had a $Q$ of $20000 \pm 4 \%$ when the impedance was matched. The loaded $Q, Q_{L}$, of the resonator can be expressed as $Q_{L}^{-1}=Q_{e}^{-1}+Q_{U}^{-1}$, where $Q_{e}$ and $Q_{U}$ are the external and unloaded $Q$, respectively. The external $Q$ is the effective $Q$ of the loading produced by the external circuitry and the unloaded $Q$ is 
the intrinsic $Q$ of the actual resonator which, in the case of the HTS coil, is determined primarily by losses due to the substrate. Since the external and unloaded $Q$ s are equal when the resonator is matched, this means that the unloaded $Q$ of the resonator is about 40000 . Fig. 2 shows the response of the HTS coil. The room temperature copper coil had a loaded $Q$ of $150 \pm 4 \%$.

The HTS coil and copper coil resonated at frequencies within a hundred kilohertz of their designed resonant frequency. The tuning rod was then used for fine tuning about the resonant frequency. Initially, difficulties were encountered with the frequency stability of the resonator but these difficulties were largely overcome by operating the resonator at temperatures well below $T_{C}$. At temperatures'slightly below $T_{C}$ small temperature fluctuations result in large changes of the resonant frequency but at lower temperatures, the resonant frequency is less sensitive to temperature fluctuations. In addition, measures were taken to dampen mechanical vibrations in order to reduce microphonics.

The $Q$ was found to be highly dependent on the sodium concentration of the phantom. The $30-\mathrm{mM}$ sodium phantom used in these studies reduced the $Q$ of the HTS coil to about $2500 \pm 4 \%$. This is an unavoidable loss in MRI of conductive bodies.

The $Q$ values did not change significantly when the HTS coil was placed in the imager. This is partly due to the HTS coil being situated parallel to the magnetic field component but this also indicates that the HTS film is of high quality with few grain boundaries.

\section{IMAGING EXPERIMENTS}

All experiments were performed using a 3-T, 6-in bore magnet. Two-dimensional Fourier imaging experiments were run on sodium phantoms with a repetition time of $100 \mathrm{~ms}$, spin echo time of $6 \mathrm{~ms}$ and four averages. The receiver bandwidth was $5 \mathrm{kHz}$. Sodium phantoms were imaged with the room temperature copper coil and HTS receiver coil described above. The image intensity profiles acquired are shown in Fig. 3(a) and (b) for the identical acquisition parameters. The phantom consisted of two sealed chambers both containing a $30-\mathrm{mM}$ $\mathrm{NaCl}$ solution separated by a 6-mm-thick Plexiglas dividing wall. The HTS coil image profile, shown in Fig. 3(a), has a very low noise floor and clearly shows the dividing wall of the phantom. The copper coil image profile, shown in Fig. 3(b), suffers from the addition of substantial noise. A quantitative measurement of the image intensity profiles found the SNR was increased by a factor of ten for the HTS coil. Remembering the relationship between SNR and imaging time, this means that the imaging time could be decreased by a factor of 100 .

\section{CONCLUSION}

We have designed and constructed an HTS receiver coil system for sodium imaging. The HTS coil system was described and the results from sodium imaging experiments were presented. The image profiles obtained demonstrate the significant SNR gains that can be achieved by using HTS coils at this frequency and coil size. The gains afforded by HTS coils can alternatively be used to decrease the imaging time. Reduced imaging time is particularly important for in vivo sodium imaging which often relies on extensive time averaging to obtain the necessary SNR. Refinements in the HTS coil system, such as reducing other noise sources, should yield further improvements in the SNR.

\section{ACKNOWLEDGMENT}

The authors are grateful to Dr. R. Withers for many helpful discussions. They also thank Dr. Y. Yang, Mr. B. Thomas, Dr. E. S. Yang, and Dr. P. O. Alderson for their assistance.

\section{REFERENCES}

[1] H. C. Seton, D. M. Bussell, and J. M. S. Hutchison, "A liquid heliumcooled RF coil and DC SQUID amplifier for MRI at $0.01 \mathrm{~T}$," in Proc. Soc. Magn. Reson., 1995, vol. 2, p. 959

[2] A. S. Hall, B. Barnard, P. McArthur, D. J. Gilderdale, I. R. Young, and G. M. Bydder, "Investigation of a whole-body receiver coil operating at liquid nitrogen temperatures," Magn. Reson. Med., vol. 7, p. 230, 1988.

[3] J. Granot, "Sodium imaging by gradient reversal," J. Magn. Reson., vol. 68 , pp. $575-581,1986$

[4] K. J. Jung, J. Katz, L. M. Boxt, S. K. Hilal, and Z. H. Cho, "Breakthrough of single-quantum coherence and its elimination in doublequantum filtering," J. Magn. Reson., vol. B107, pp. 235-241, 1995.

[5] R. Reddy, M. Shinnar, Z. Wang, and J. S. Leigh, "Multiple-quantum filters of spin-3/2 with pulses of arbitrary flip angle," J. Magn. Reson., vol. B104, pp. 148-152, 1994.

[6] S. K. Hilal, C. H. Oh, I. K. Mun, and A. J. Silver, "Sodium imaging," in Magnetic Resonance Imaging, D. D. Stark and W. G. Bradley, Eds. St. Louis, MO: Mosby-Year Book, 1992, pp. 1091-1110.

[7] N. Bansal and V. Seshan, "Three-dimensional triple-quantum-filtered ${ }^{23} \mathrm{Na}$ imaging of rabbit kidney with weighted signal averaging," in Proc. Soc. Magn. Reson., 1995, vol. 2, p. 1204

[8] R. D. Black, T. A. Early, P. B. Roemer, O. M. Mueller, A. MogroCampero, L. G. Turner, and G. A. Johnson, "A high-temperature superconducting receiver for nuclear magnetic resonance microscopy," Sci., vol. 259, pp. 793-795, 1993.

[9] R. D. Black, T. A. Early, and G. A. Johnson, "Performance of a hightemperature superconducting resonator for high-field imaging," J. Magn. Reson., vol. A113, pp. 74-80, 1995.

[10] M. Yap, R. D. Black, W. W. Brey, B. F. Cole, M. E. Johansson, G. A. Johnson, V. Y. Kotsubo, R. Nast, and R. S. Withers, "High temperature superconducting probe for NMR microscopy," in Proc. Soc. Magn. Reson., 1995, vol. 2, p. 958.

[11] A. Lauder, C. Wilker, D. J. Kountz, W. L. Holstein, and D. W. Face, "High performance superconducting thin films on large area substrates," IEEE Trans. Appl. Superconduct., vol. 3, pp. 1683-1686, 1993.

[12] R. S. Withers, G. C. Liang, B. F. Cole, and M. Johansson, "Thin-film HTS probe coils for magnetic resonance imaging," IEEE Trans. Appl. Superconduct., vol. 3, pp. 2450-2453, 1993.

[13] J. G. van Heteren, T. W. James, and L. C. Bourne, "Thin film high temperature superconducting RF coils for low field MRI," Magn. Reson. Med., vol. 32, pp. 396-400, 1994.

[14] A. Raad and L. Darrasse, "Optimization of NMR receiver bandwidth by inductive coupling," Magn. Reson. Imag., vol. 10, pp. 55-65, 1992. 